\title{
MARCO JURÍDICO-CONCEPTUAL DEL SISTEMA PÚBLICO DE LOS SERVICIOS SOCIALES
}

\section{LEGAL AND CONCEPTUAL FRAMEWORK OF THE PUBLIC SYSTEM OF SOCIAL SERVICES}

\author{
Saida Ayala García \\ Universidad de Cádiz. Cádiz/España \\ saida.ayala@.gmail.com
}

Recibido/Received: 18/05/2018

Modificado/Modified: 15/09/2018

Aceptado/Accepted: 22/09/2018

\section{RESUMEN}

En el marco del Sistema Público de Servicios Sociales, esta publicación realiza un estudio descriptivo, analítico y propositivo de los orígenes, evolución, y régimen competencial de estos servicios. Este estudio pretender ser una fuente de conocimiento en esta materia, para ello se analizarán conceptos tan relacionados como diferentes: la "caridad", la "beneficencia", la "Seguridad Social", la "asistencia social", la "protección social", el "trabajo social" y los "Servicios Sociales", con el objetivo principal de redefinir de manera eficaz y eficiente los distintos sistemas de protección social.

\section{PALABRAS CLAVE}

Estado Social; Servicios Sociales; Seguridad Social; asistencia social; beneficencia.

\section{SUMARIO}

1. Introducción. 2. Definición y diferencias entre Asistencia Social, Servicios Sociales y Seguridad Social. 3. De la Asistencia Social a los Servicios Sociales. 4. Asistencia Social y Protección Social. 5. ¿Qué es el Sistema Público de Servicios Sociales? 6. Conclusiones. Bibliografía.

\begin{abstract}
Within the framework of the Public Social Services System, this paper carries out a descriptive, analytical and proactive study of the origins, evolution, and competence regime of these services. This study aims to be a source of knowledge in this matter analyzing concepts as related as different, for instance: "charity", "Social Security", "social assistance", "social protection", "social work" and "Social Services", with the main objective of redefining effectively and efficiently the different social protection systems.
\end{abstract}

\section{KEYWORDS}

Social Services; Social State; Social Security; Social Assistance; Charity.

\section{CONTENTS}

1. Introduction. 2. Definition and differences between Social Assistance, Social Services and Social Security. 3. From Social Assistance to Social Services. 4. Social Assistance and Social Protection 5. What is the Public Social Services System? 6. Conclusions. References. 


\section{INTRODUCCIÓN}

La evolución de términos utilizados en el sistema de Servicios Sociales (servicios de caridad, beneficencia...) es una manifestación evidente de su gran desarrollo normativo, organizativo y competencial, si bien se trata de una expresión que todavía no está plenamente consolidada en el ordenamiento jurídico. Esto afecta, junto a las restricciones presupuestarias aprobadas desde 2012 hasta la actualidad como consecuencia de la crisis económica (20072017) y de la reforma constitucional de 2011, a que los Servicios Sociales públicos, ni trate de raíz los problemas en presencia ni satisfagan de manera efectiva las demandas de los usuarios.

Partiendo del concepto de servicio público, para calificar los Servicios Sociales deben diferenciarse los servicios públicos económicos, de los servicios públicos no económicos o sociales. Mientras los primeros son aquellos servicios públicos que satisfacen necesidades estrechas y directamente relacionadas con la actividad económica, los Servicios Sociales se puede decir que son servicios no económicos porque la actividad prestacional de la administración pública tiene por objeto inmediato a las personas como tal y como usuario del servicio, como ocurre con la sanidad, la educación, la cultura (Fantov, 2008: 16-17).

Además, si bien los servicios públicos pueden ser uti universi - prestaciones de interés general que los ciudadanos reciben del Estado derivan del ejercicio de funciones conectadas directamente a la soberanía, como la Justicia, la defensa nacional, la seguridad pública o las relaciones internacionales-, en el caso de los Servicios Sociales se trata de servicios públicos uti singuli, definidos como aquellos que se disfrutan individualizadamente por los usuarios, quienes puedan asumir todo o parte de su coste.

Sobre la clasificación de los servicios públicos en régimen de libre concurrencia y servicios públicos reservados, en el caso de los Servicios Sociales, la asunción de una actividad como servicio público no excluye la realización de actividades semejantes y concurrentes por las empresas o entidades privadas. No se trata, pues, de un servicio público reservado.

Además, en la clasificación de servicios públicos obligatorio y no obligatorio, los Servicios Sociales son obligatorios por así preverlo la Constitución de 1978 y las leyes sectoriales sobre la materia, principalmente las leyes autonómicas, como la Ley 9/2016, de 27 de diciembre, de Servicios Sociales de la Comunidad Autónoma de Andalucía.

Pues bien, este artículo, pretende esclarecer conceptos como "caridad", "beneficencia", "Seguridad Social", "asistencia social", "protección social”, "Servicios Sociales"..., además de servir para mostrar lo que ha cambiado el sistema - aunque aún quede labor por hacer-, y tomar conciencia sobre el recorrido realizado hasta llegar a lo que actualmente se conoce por Servicios Sociales.

En cuanto a la metodología utilizada, se han utilizado las técnicas interpretativas y analíticas propias las Ciencias Sociales y de la Ciencia Jurídica.

\section{DEFINICIÓN Y DIFERENCIAS ENTRE ASISTENCIA SOCIAL, SERVICIOS SOCIALES Y SEGURIDAD SOCIAL}

Históricamente, se utilizaba el término de "caridad" y "beneficencia", caracterizados por la atención a los "pobres". Este ha sido el término ampliamente utilizado en las fuentes en el siglo XVIII y, como forma organizativa de la atención social, fue dominante hasta buena parte del siglo XX, quedando muy arraigado en el sistema de asistencia social hasta la 
irrupción de los sistemas privados de mutualidad, principalmente para trabajadores no asalariados, y los sistemas públicos de "previsión social" (Santos, 2012: 97), que se caracterizaban por la atención exclusiva a los trabajadores asalariados y a sus familiares, en el contexto de las relaciones industriales y de servicios. La actividad profesional es inherente a la previsión social, que principalmente amparaba a los trabajadores asalariados.

Pues bien, de la previsión social se pasó a la Seguridad Social como modelo cada vez más evolucionado en su dimensión subjetiva (se pasa de un conjunto de personas protegidas que han cotizado, en el caso de las prestaciones contributivas, hasta su universalización y la regulación de las pensiones no contributivas) y su dimensión objetiva (contingencias), y siempre por imperativo u obligación de la ley (Vicente, 2015:131-157).

Hay autores como Pemán, que defienden el mantenimiento de la expresión "asistencia social", pues sostiene que cualquier "noción más amplia" es de "perfiles difusos y cuyos contornos no es posible acotar de forma rigurosa" y ella cabe incluir las "diversas actuaciones públicas encaminadas a paliar las situaciones de necesidad mediante el otorgamiento de prestaciones, ayudas o ventajas que implican una compensación de cargas o de circunstancias específicas" que inciden sobre determinados colectivos de personas, sin subordinación al requisito de que los "beneficiarios acrediten que se encuentran por debajo de un determinado nivel de renta"(Pemán, 2005: 271-272).

Estas técnicas públicas de protección social quedan reservadas con carácter exclusivo a las Comunidades Autónomas en el orden normativo, tal como permite el art. 148.1.20 CE y han hecho efectivo los distintos Estatutos de Autonomía. Esta doctrina sobre el objeto de la materia "asistencia social" ha sido después recogida por nuestras SSTC 33/2014, de 27 de febrero, FJ 4, y 18/2016, de 4 de febrero, FJ 7 b).

Por otro lado, se puede afirmar, como lo hace Rodríguez, en su tercera ponencia sobre el concepto de asistencia social, que el Sistema de la Seguridad Social es el mejor mecanismo protector contra riesgos sociales que se conoce, aunque con limitaciones presupuestarias y que el sistema de asistencia social y de Servicios Sociales presenta un carácter adicional respecto a este (Rodríguez: 5-6). En efecto, el trabajo es un ámbito de socialización pero, sobre todo, un recurso económico que provee de los medios para satisfacer las necesidades económicas. Por este motivo, la Seguridad Social ha previsto una serie de contingencias en el contexto de una relación laboral (prestaciones sanitarias...) pero también subsanar la escasez de recursos (alimentos, vivienda...) derivada de la falta de trabajo. Solo cuando las situaciones de necesidad no pueden resolverse exclusivamente desde el Sistema de Seguridad Social, que presenta innumerables limitaciones, los poderes públicos deben procurar al individuo los recursos necesarios para su subsistencia ante situaciones de necesidad.

Pero se ha de tener en cuenta que el ámbito subjetivo del Sistema de Seguridad Social no comprende a todas las situaciones en estado de necesidad, y por tal motivo, la asistencia social tiene como destinatarios a las personas no comprendidas en el ámbito de protección o de riesgos cubiertos por la Seguridad Social (Rodríguez: 11).

Mientras la Seguridad Social se rige básicamente por el criterio subjetivo o mixto subjetivo-objetivo (sujetos y contingencias) y protege universalmente a los sujetos comprendidos en el ámbito de las contingencias típicas, la asistencia social no se articula subjetivamente a partir de los destinatarios, sino que objetiva y territorialmente. Esto es, objetivamente porque determina los riesgos y protege a quien se encuentre en situaciones de vulnerabilidad, y territorialmente porque, a tratarse de una competencia de gran desarrollo autonómico, depende de la configuración estatutaria y legislativa de cada Comunidad Autónoma: ingreso mínimo o renta mínima de inserción a las personas que acrediten una situación de debilidad económica, redes de Servicios Sociales para la atención para personas 
con discapacidad, infancia y adolescencia, familias, personas en situación de exclusión social o marginación, como los liberados de prisión; extranjeros y minorías étnicas...

Sobre este principio general de delimitación, existen prestaciones en el campo de aplicación de la Seguridad Social que son prestaciones de Servicios Sociales, casi siempre coyunturales y no consolidables, y en muchos casos la asistencia social complementa los riesgos protegidos por la Seguridad Social.

Un espacio de particular interés presentan las prestaciones no contributivas, que ampliaron el ámbito de aplicación del sistema de Seguridad Social más allá de la contributividad imperante hasta entonces, las prestaciones no contributivas no cumplen de forma total el proceso de universalización: ni protegen de todos los riesgos, sino que responden a contingencias (edad e incapacidad) ya cubiertas en la modalidad contributiva; ni superan la concepción de las prestaciones familiares dirigidas a apoyar económicamente el exceso de gastos específicos (farmacéuticos, hijos, primera necesidad...). Por ahora son solo un remedio que subsana solo parcialmente y de forma fragmentaria las deficiencias concretas del modelo contributivo (Pérez, 2003: 105-118).

\subsection{Delimitación Constitucional entre asistencia social, Servicios Sociales y Seguridad Social}

Concentrándonos en la de limitación constitucional, se puede decir que en su formulación concreta, el fundamento constitucional de esta actividad prestacional lo encontramos en el Capítulo III del Título I de la Constitución (familia del art. 39, salud del art. 43, minusvalía del art. 49 y vejez del art. 50). Y en su formulación genérica, lo hallamos en el art.1.1. (Estado social), en la dignidad de la persona (art. 10.1 CE) y en el art. 9.2 CE, donde se establece una actividad promocional de los poderes públicos y el principio de igualdad real, una de cuyas manifestaciones es la igualdad "en el punto de llegada", como complemento de la "igualdad en el punto de salida" (Rey, 2014: 25-28) como contenido de gran relevancia en periodos de crisis del Estado social. En principio, esa tutela puede provenir del Sistema de Seguridad Social o de cualquier otra competencia pública: asistencia social, dependencia...

Afirman Alonso y Gonzalo, que lo deseable sería un Sistema de Seguridad Social que cubriera todas las contingencias posibles e hiciera innecesaria la asistencia social y los Servicios Sociales, aunque reconocen que siempre "existirán fenómenos de exclusión social a los que atender, y que no están tipificados como de Seguridad Social (2000)".

Aunque en principio existe una delimitación constitucional entre la asistencia social y los Servicios Sociales, por un lado, y la Seguridad Social, por otro -el art. 148.1.20 CE y el principio constitucional de caja única, respectivamente- (De la Villa, 2003:591-594), la generalizada asunción competencial autonómica de la asistencia social ha llevado a invadir o complementar, según los casos, el ámbito competencial de la Seguridad Social para atender las necesidades no cubiertas por el Sistema de Seguridad Social, y en otras complementando a este.

En sentido contrario, la esfera competencial autonómica también ha sido objeto de invasión por parte de la legislación de Seguridad Social, particularmente en el caso de las prestaciones no contributivas o la renta activa de inserción, lo que se denomina "Seguridad Social no contingencial", que es una zona gris común que concurre con la asistencia social. Por tal motivo, un sector doctrinal ha defendido que la Seguridad Social se defina de forma meramente "contingencial", para diferenciarla de la asistencia social, como acción pública acontingencial justificada por una situación de necesidad (Hurtado, 1993: 472-473).

$\mathrm{Y}$, en el caso de los modelos mixtos, que combinan prestaciones contributivas y no contributivas, se trataría del ámbito de la Seguridad Social (Rodríguez-Piñero \& Bravo- 
Ferrer, 1988:4; Maldonado, 1999: 521 y ss.).

Así concebido, el fundamento constitucional de la protección social a través de la Seguridad Social en sentido amplio se encuentra en el art. $41 \mathrm{CE}$ que atribuye a los poderes públicos el mandato y la competencia sobre "un régimen público de Seguridad Social para todos los ciudadanos, que garantice la asistencia y prestaciones sociales suficientes ante situaciones de necesidad, especialmente en caso de desempleo". Además, este precepto indica que "la asistencia y prestaciones complementarias serán libres".

Por otra parte, el art. 129 CE preceptúa que "la ley establecerá las formas de participación de los interesados en la Seguridad Social”. Y en el ámbito competencial, el art. 149.1.17 atribuye al Estado la competencia exclusiva en materia de "legislación básica y régimen económico de la Seguridad Social, sin perjuicio de la ejecución de sus servicios por las Comunidades Autónomas". Con esto se quiere decir que, la STC 76/1986 y la STC 239/2002 avalan que la asistencia social "tiene un espacio constitucionalmente reservado, pero no un contenido mínimo". Pero bien es sabido que existe una gran dificultad para "deslindar con nitidez los conceptos de Seguridad Social y asistencia social" (Rodríguez, 2011: 1 en AA.VV, 1980: 433-436), principalmente porque el propio concepto de Seguridad Social es público (no privado, frente al sistema de mutualidades) y "coyuntural tanto en el tiempo como en el espacio". Y, aunque la Constitución de 1978 y los Estatutos de Autonomías los regulan parcialmente, no diferencian entre Servicios Sociales, asistencia social y Seguridad Social (De la Villa, 1981: 18-19; De Vicente, 2002: 9-12), lo que exige una delimitación en las leyes sectoriales reguladoras de esta materia que son estatales y autonómicas dependiendo del bloque de la constitucionalidad existente en estas materias.

El Sistema de Seguridad Social protegería en la dimensión horizontal a unos determinados sujetos (1) de unas contingencias típicas (2) a través de un catálogo de naturaleza coyuntural, tutelando en su dimensión vertical hasta el nivel legalmente establecido (3). Fuera de esas tres dimensiones que configuran y agotan el Sistema de Seguridad Social, actuaría la asistencia social. Caso particular presente el ámbito donde se inserta la renta básica o la renta mínima garantizada como recurso mínimo alternativo o complementario de la asistencia social. Hasta ahora, la atención a esos sujetos en especial estado de necesidad tiene lugar por la vía de la asistencia social. La Seguridad Social y la asistencia social se relacionan así sobre la base de la complementariedad y de la suplementariedad, porque la asistencia social completa el ámbito subjetivo de aquella mediante la dispensación de beneficios a quienes carecen de recursos y no cumplen los requisitos para causar una prestación de Seguridad Social.

Si en ocasiones la asistencia social ocupa el ámbito material donde no llega la acción protectora de la Seguridad Social, en otras releva al propio Sistema en la protección de una contingencia cuyas consecuencias no han podido ser completamente superadas pese a la dispensación de la acción protectora propia de la Seguridad Social. Por lo tanto, el ámbito objetivo y subjetivo de la asistencia social queda así estrechamente vinculado al Sistema de Seguridad Social, complementando o supliendo a este en situaciones de necesidad caracterizadas, bien por la debilidad económica extrema, bien por la protección a colectivos cuya necesidad es cualificada por diversas circunstancias: discapacidad, maltrato, desempleo de larga duración...

\section{DE LA ASISTENCIA SOCIAL A LOS SERVICIOS SOCIALES}

La asistencia social está prevista como materia competencial en el art. 148.1. 20 $\mathrm{CE}$, con 
el fin de atribuir la competencia a las CCAA. Por su parte, el art. 50 CE prevé un mandato a los poderes públicos, destinado a promover el bienestar de los ciudadanos durante la tercera edad "mediante un sistema de Servicios Sociales que atenderán sus problemas específicos de salud, vivienda, cultura y ocio". En esta regulación, como afirmó tempranamente De Otto, la palabra Constitución y, con ella, la expresión "Derecho Constitucional (2008)" refleja el avance terminológico desde la "asistencia social" a los "Servicios Sociales", que sustituye el término de la beneficencia, empleado secularmente para designar la actividad pública o privada que satisfacía las necesidades sociales.

El significado de asistencia social, se refiere a "la acción de prestar socorro, favor, ayuda" (Santos, 2012:100). No es esa la labor que desempeñan los trabajadores sociales, ni tampoco la práctica que se realiza desde los Servicios Sociales... No es una simple "ayuda", sino como sostiene Martín y Alario (2016), es una actividad profesional cargada de conocimientos técnicos y científicos.

Según su configuración jurisprudencial, la STC 239/2002, de 11 de diciembre, define la asistencia social como un complemento a las pensiones no contributivas de jubilación e invalidez concedido por la Junta de Andalucía (Álvarez \& Pérez, 2005:140 y ss.; Molina, 2005:38-39). Además, "las competencias autonómicas sobre materias no incluidas en el art. 149.1 CE, aunque se enuncien como 'competencias exclusivas', no cierran el paso a las competencias estatales previstas en aquel precepto constitucional" (STC 33/2014, de 27 de febrero, FJ 4).

No obstante, aunque la asistencia social prestada en el marco de los sistemas autonómicos de Servicios Sociales no cubre ni mucho menos todo el extenso campo material de la asistencia social, "puede considerarse que constituye en la actualidad el núcleo más característico o prototípico de la misma” (Pemán, 2005:45).

\section{ASISTENCIA SOCIAL Y PROTECCIÓN SOCIAL}

Junto a los términos Seguridad Social, asistencia social, Servicios Sociales o previsión social, algunas normas se refieren a la expresión "protección social", sin contenido propio ni carácter excluyente de los demás.

La protección social, se trata de un nomen iuris genérico que comprende las distintas técnicas a través de las cuales los poderes públicos atienden las situaciones de necesidad, principalmente la Seguridad Social y la asistencia social (Persiani, 1997:22-24). No obstante, este término genérico ha permitido al Estado eludir el término "asistencia social" en el caso de ámbitos que han requerido la actuación del legislador estatal, por ejemplo en el caso de las ayudas previstas en el art. 27 LO 1/2004, de 28 de diciembre, de Medidas de Protección Integral contra la Violencia de Género, que tampoco se califican como Seguridad Social, pese a que guardan un paralelismo evidente con algunas prestaciones del Sistema. La invocación de la "protección social" permitiría la actuación del Estado. Por ejemplo, según el F.J. $3^{\circ}$ de la STC 18/2017, de 2 de febrero de 2017, el establecimiento de un sistema de autorizaciones especiales para el estacionamiento de los vehículos de personas con limitaciones físicas para desplazarse constituye una medida de protección social, porque les proporciona un beneficio en atención a sus circunstancias personales de desventaja respecto al resto de la colectividad. Ahora bien, no toda medida pública de contenido social o protectora de grupos desfavorecidos constituye de por sí asistencia social en sentido competencial. En otras palabras, no sólo las Administraciones públicas con competencias formales de asistencia social pueden adoptar medidas sociales o beneficiosas de colectivos 
desfavorecidos, dado que la cláusula del Estado social y su corolario en el artículo 9.2 son transversales y precisamente deben proyectarse en todos los ámbitos de la sociedad y del ordenamiento jurídico por voluntad del constituyente.

\section{5. ¿QUÉ ES EL SISTEMA PÚBLICO DE SERVICIOS SOCIALES?}

El término de Servicios Sociales está estrechamente vinculado a la asistencia social y hace referencia a los aspectos organizativos de ésta, que se identificaría con los aspectos funcionales y materiales. Como expresión "Servicios Sociales", aparece y se consolida en el siglo $\mathrm{XX}$, aunque la sociedad civil está algo difusa en cuánto al término de estos servicios, $\mathrm{y}$ uno de los motivos puede ser la variedad de denominaciones empleadas por las Administraciones Públicas para señalar el departamento competente para gestionar esta materia (Asuntos Sociales, Políticas Sociales, Servicios Sociales, Bienestar Social...), incluso encuadrarla en otras rúbricas genéricas (Trabajo, Igualdad, Empleo, S.S...) (Santos, 2012:26).

Aludiendo a la Ley 9/2016, de 27 de diciembre, de Servicios Sociales de Andalucía, la configuración legislativa en España se articula en dos niveles:

1. Servicios Sociales Generales, Comunitarios o de Atención Primaria destinados a toda la población a través de prestaciones básicas y específicas. Estos servicios se gestionan a través de los centros de Servicios Sociales y a través de centros comunitarios (centros de acogida, centros de día o centros ocupacionales) de las Corporaciones Locales.

2. Servicios Sociales Especializados, que están dirigidos a colectivos específicos de población (tercera edad, infancia, juventud, toxicómanos, personas sin hogar, etc.), y se relacionan con las necesidades personales y características grupales de los usuarios: centros de día para adultos, centros de día para menores, residencias, centros ocupacionales y centros de protección y residenciales, centros de inserción social, centros para personas discapacitadas, entre otros. La competencia de gestión corresponde a la Administración Autonómica y Local. Orgánicamente, el Gobierno de la Nación adscribe los Servicios Sociales a la área de actuación orgánica dentro del Ministerio de Sanidad y Servicios Sociales, pero, sin embargo, para el Consejo de Gobierno de la Junta de Andalucía estos servicios están enmarcados dentro de la Consejería de Igualdad y Políticas Sociales, con abandono del término de Servicios Sociales. Por otro lado, estos servicios en varias Diputaciones Provinciales de Andalucía se engloban dentro del apartado de Igualdad y Bienestar Social. Por último, si se refiere a los Ayuntamientos, muchos de ellos reseñan a este departamento como Servicios Sociales, pero muchos otros aún siguen utilizando el término de "Asuntos Sociales" o de "Bienestar Social".

Para Pemán, a favor de la asistencia social, resulta "razonable mantener la continuidad de una expresión que goza de un claro arraigo en nuestra tradición legislativa y doctrinal (2005)". Sin embargo, siguiendo a Santos, y atendiendo a lo que ya establecen el bloque de la constitucionalidad en los Estatutos de Autonomía, e igual que en otros ámbitos del servicio público (sanidad, educación...), parece conveniente consolidar el término de Servicios Sociales, y que éste sea usado de la misma manera tanto a nivel institucional como en todas las estructuras administrativas que se hacen cargo de esta competencia: Ayuntamientos, Diputaciones Provinciales, Concejalías, Departamentos Autonómicos, Direcciones Generales, Consejerías etc. (Pemán, 2005:102).Sólo de esta manera se podrá definir estos servicios en el marco de un sistema homogéneo. 


\section{CONCLUSIONES}

Tras el análisis de los datos que se han ido estudiando, este artículo finaliza con cuatro conclusiones:

En primer lugar, se ha constatado la evolución experimentada, y solo parcialmente asumida por las Administraciones públicas en presencia, por el marco jurídico y conceptual sobre los Servicios Sociales. Los Servicios Sociales se han consolidado y las reformas legislativas han sido muchas y relevantes, pero no se ha avanzado al mismo ritmo en las infraestructuras sobre servicios sociales, ni en su financiación, ni siquiera en la planificación o la formación del personal.

En segundo lugar, se confirma que los primeros problemas se plantean, a nivel general, en el ámbito conceptual. Aunque la expresión "asistencia social" es aún un término admitido y así lo utiliza en sus sentencias más recientes el Tribunal Constitucional como supremo intérprete de la Constitución, e incluso ocasionalmente el legislador, lo cierto es que el Estatuto de Autonomía de Andalucía, otros Estatutos de Autonomía y la legislación autonómica sectorial llevan diez años empleando el término aceptado en las Ciencias Sociales: "Servicios Sociales". Esta dualidad terminológica ha tenido como consecuencia el uso de la "asistencia social" en la doctrina jurisprudencial a partir de la regulación del art. 148.1. 20a CE, que tiene ya casi cuatro décadas. En España, las necesidades sociales de las personas se gestionan siguiendo un marco conceptual actualizado (Servicios Sociales), pero sus problemas jurídicos y competenciales se resuelven atendiendo a un concepto caduco que ya no se sigue en los países de nuestro contexto político y económico: asistencia social.

Este doble marco conceptual, que desde hace tres décadas genera incertidumbre entre los sujetos en presencia y conflictividad competencial entre las Administraciones Públicas, exige una delimitación las competencias entre el Estado y las Comunidades Autónomas. La regulación de los Servicios Sociales sigue perteneciendo nominalmente al ámbito material de la "asistencia social", lo que a futuro exige una actualización de la normativa española sobre esta materia, empezando por el texto de la Constitución de 1978 (Capítulo III del Título I), que en una futura reforma debería adoptar los principios de universalidad, generalidad e imperatividad de los Servicios Sociales, frente al carácter particular y discrecional de la asistencia social.

En tercer lugar, al examinar el más actualizado marco conceptual sobre la "asistencia social", es evidente que tanto su invocación constitucional como jurisprudencial ha quedado obsoleta y poco definida. Así pues, se debería llevar a cabo una futura reforma al marco constitucional donde se incluyan conceptos más actualizados como el "Sistema Público de Servicios Sociales" y "Servicios Sociales de Interés General", relativos a un conjunto de órganos que prestan unas prestaciones para garantizar la protección social de las personas y mejorar la calidad de vida, garantizando y favoreciendo valores recogidos en la Constitución como la igualdad (art. 1.1), la dignidad (art. 10.1), la justicia (art. 1.1) haciendo progresar en el "Estado social y democrático de Derecho" hacia la consecución de la sociedad democrática avanzada que proclama el Preámbulo constitucional, el Derecho de la Unión Europea y los tratados internacionales referidos en el art. 10.2 CE (art. 25 de la Declaración de los Derechos Humanos, art. 14 de la Carta Social Europea...). Y, como consecuencia de lo anterior, queda pendiente también la definición de un marco completo de relaciones entre las Administraciones públicas y las entidades de iniciativa social (tercer sector), que la Ley 9/2016 simplemente contempla en el ámbito de los de cooperación y coordinación, impidiendo la realización de funciones sociales con fines y objetivos públicos a las entidades privadas. 
Una cuarta conclusión merecen las relaciones entre los ámbitos competenciales de los Servicios Sociales, la Seguridad Social y la protección social, cuya compleja casuística ha condicionado de forma intensa la regulación, planificación y gestión de los Servicios Sociales a nivel local. A los efectos de su futura planificación debe tenerse en cuenta, por una parte, que la protección social se refiere a los distintos sistemas a través de las cuales los poderes públicos atienden las situaciones de necesidad, pero entendiendo que no toda acción protectora constituye asistencia social en sentido competencial. Por otra parte, también se plantea la antigua, aunque mejor estudiada doctrinalmente, delimitación objetiva entre los Servicios Sociales y la Seguridad Social (art. $149.117^{\text {a }} \mathrm{CE}$ ). Debido a la tradicional configuración de las competencias vinculadas al Estado social, a la hora de planificar los Servicios Sociales existe una zona común compartida con la Seguridad Social. Por lo tanto, aunque la jurisprudencia constitucional ha delimitado algunos de sus casos más problemáticos, subsiste la necesidad de una mejor diferenciación normativa entre los Servicios Sociales y la Seguridad Social, que son dos pilares fundamentales para cubrir las contingencias sociales que en el momento presente están abandonados a las extralimitaciones competenciales (casi siempre a favor del Estado), las duplicidades administrativas y la confusión ciudadana, impidiendo una adecuada coordinación de las estructuras administrativas y planificación eficiente de su realidad y financiación.

Hay que tener en cuenta que tanto los Servicios Sociales de atención primaria, como los Servicios Sociales de atención especializada, actúan a partir de un título competencial de asistencia social que se plasma en los catálogos y carteras de prestaciones y servicios de las Comunidades Autónomas. En principio, la Seguridad Social, aunque puedan incluir medidas de carácter asistencial, se encarga de las prestaciones contributivas o no contributivas, dejando fuera de su ámbito protector las prestaciones de carácter público cuyo objetivo fuese complementar o mejorar las prestaciones no contributivas. Pero no siempre es así, y en la medida en que aún no está delimitado legalmente hasta qué punto deben o pueden intervenir los Servicios Sociales o la Seguridad Social en cada asunto, se hace necesaria, como se ha explicado, una clara delimitación constitucional y legal de la "Seguridad Social" y los "Servicios Sociales" donde se recojan de manera precisa el tipo de prestación, los títulos competenciales, los servicios, los destinatarios y sus competencias.

En suma, se propone la redefinición del Sistema Público de Servicios Sociales (estatal), tanto en el ámbito financiero como competencial, una mayor coordinación de los Servicios Sociales autonómicos y locales, y un mayor compromiso normativo y administrativo en la calidad de las prestaciones gestionadas por el tercer sector. Además, sin perjuicio de la competencia que corresponde a la Junta de Andalucía y a los entes locales, el Estado también debe asumir su responsabilidad en la gestión de estos servicios en cumplimiento de los mandatos constitucionales bajo el título competencial del art. 149.1. $1^{\mathrm{a}} \mathrm{CE}$, con el fin de hacer realidad unos servicios universales y públicos que son contenidos esenciales del Estado Social proclamado en el art. 1.1 CE y el mandato promocional del art. 9.2 CE.

\section{BIBLIOGRAFÍA}

Alemán Bracho, C. y Fernández García, T. (2008) Introducción a los Servicios Sociales. Madrid: Universidad Nacional de Educación a Distancia (UNED).

Alonso Seco, J.M. y Gonzalo González, B. (2000) La asistencia social y los servicios sociales en España. Madrid: BOE.

Álvarez Cortés, J.C. y Pérez Yáñez, R. (2005) “Algunas novedades recientes en la legislación de 
Seguridad Social", $R L, 15-16: 140$ y ss.

De la Villa Gil, L.E. (1981) "Potestades normativas de las Comunidades Autónomas en materia laboral y de Seguridad Social", $D L, 1: 18-19$.

De La Villa Gil, L.E. (2003) "El contenido constitucional de la asistencia social", en A. Montoya Melgar, El trabajo y la Constitución, Madrid: MTAS, pp. 591-594.

De Otto, I. (2008) Derecho Constitucional. Sistema de fuentes, Ariel, Barcelona.

De Vicente Pachés, F. (2002) "Asistencia social y Servicios Sociales: Régimen de distribución de competencias", Tribuna Social, 138: 9-12

Fantov, F. (2008) Sistemas públicos de servicios sociales. Bilbao: Universidad de Deusto, en: https://goo.gl/qsr86T (consultado 10/08/2018).

Hurtado González, L. (1993) “Asistencia social y Seguridad Social: sus fronteras actuales”, Actualidad Laboral, 25: 472-473.

Maldonado Molina, J.A. (1999) "La descentralización territorial y las pensiones no contributivas. A propósito del Decreto 284/1998, de 29 de diciembre, del Consejo de Gobierno de la Junta de Andalucía”. Revista Actualidad Laboral, 28(2): 521 y ss.

Martín Pérez, E. y Alario Bancells, R. (2016) "La sistematización como "proceso sine qua non" en la reformulación y regeneración del quehacer profesional de los servicios sociales", en: https://goo.gl/MZPsFn (consultado 9/9/2018).

Molina Navarrete, C. (2005) "Del «mal» e «inútil» uso del «poder legislativo» en materia de Seguridad Social: la Ley $4 / 2005$, sobre efectos en las prestaciones no contributivas de los complementos autonómicos", $A S,(12), 38-39$.

Pemán Gavín, J. (2005) "La asistencia social en España: delimitación conceptual y marco jurídico general", Documentación Administrativa, 44: 271-272 en: hyperurl.co/4llkp1_(consultado 18/09/2018).

Pérez Royo, M. R. (2003) "Seguridad Social y asistencia social en el Estado de las Autonomías". Relaciones laborales: Revista crítica de teoría y práctica, 1:105-118.

Persiani, M. (1997) Diritto della previdenza sociale, Padova: CEDAM.

Rey Martínez, F. (2014) "El principio de igualdad en el contexto de la crisis del Estado social: diez problemas actuales", Fundamentos: Cuadernos monográficos de Teoría del Estado, Derecho Público e Historia Constitucional, 8: 25-28, en: https://goo.gl/6E3oeP_(consultado 13/07/2018).

Rodríguez Cardo, I. A. (2011) "El concepto de asistencia social: un foco de permanente tensión entre el Estado y las Comunidades Autónomas", en AA.VV. Los nuevos marcos de relaciones laborales en el renovado estado de las autonomías: XXI Congreso Nacional de Derecho del Trabajo y de la Seguridad Social, pp. 797-816, en: https://goo.gl/WE9h8B_(consultado 22/8/2018).

Rodríguez-Piñero y Bravo-Ferrer, M. (1988) "Pensiones no contributivas y Seguridad Social". Relaciones Laborales, 12: 4

Santos Martí, J. (2012) El cuarto pilar: Un nuevo relato para los Servicios Sociales. Madrid: Paraninfo.

Vicente Palacio, M.A. (2015) “Crisis y Seguridad Social”. Revista Derecho social y empresa, (3): 131157, en: https://goo.gl/KrNAZR (consultado: 16/08/2018).

\section{Breve currículo:}

\section{Saida Ayala García}

Doctora en Trabajo Social por la Universidad de Cádiz. Profesora del Título de Experto en Derecho de Extranjería y de la Cooperación al Desarrollo (UCA). Investigadora del Grupo SEJ-058 PAIDI de la Universidad de Cádiz. 